DRAFT VERSION August 5, 2021

Preprint typeset using LATEX style AASTeX6 v. 1.0

\title{
EVIDENCE FOR A NEUTRAL IRON LINE GENERATED BY MEV PROTONS FROM SUPERNOVA REMNANTS INTERACTING WITH MOLECULAR CLOUDS
}

\author{
Kumiko K. Nobukawa ${ }^{1}$, Masayoshi Nobukawa ${ }^{2}$, Katsuji Koyama ${ }^{3}$, Shigeo Yamauchi ${ }^{1}$, Hideki UChiYAma ${ }^{4}$, HiRomichi \\ OKON $^{3}$, TAKAAKI TANAKA ${ }^{3}$, HIROYUKI UCHIDA ${ }^{3}$, AND TAKESHI G. TSURU ${ }^{3}$
}

${ }^{1}$ Department of Physics, Faculty of Science, Nara Women's University, Kitauoyanishi-machi, Nara, Nara 630-8506, Japan
${ }^{2}$ Faculty of Education, Nara University of Education, Takabatake-cho, Nara, Nara 630-8528, Japan
${ }^{3}$ Department of Physics, Graduate School of Science, Kyoto University, Kitashirakawa-oiwake-cho, Sakyo-ku, Kyoto, Kyoto 606-8502, Japan
${ }^{4}$ Faculty of Education, Shizuoka University, 836 Ohya, Suruga-ku, Shizuoka, Shizuoka 422-8529, Japan

\begin{abstract}
Supernova remnants (SNRs) have been prime candidates for Galactic cosmic-ray accelerators. When lowenergy cosmic-ray protons (LECRp) collide with interstellar gas, they ionize neutral iron atoms and emit the neutral iron line $(\mathrm{Fe} \mathrm{I} \mathrm{K} \alpha$ ) at $6.40 \mathrm{keV}$. We search for the iron K-shell line in seven SNRs from the Suzaku archive data of the Galactic plane in the $6^{\circ} \lesssim l \lesssim 40^{\circ},|b|<1^{\circ}$ region. All these SNRs interact with molecular clouds. We discover Fe I K $\alpha$ line emissions from five SNRs (W28, Kes 67, Kes 69, Kes 78, and W44). The spectra and morphologies suggest that the Fe I K $\alpha$ line is produced by interactions between LECRp and the adjacent cold gas. The proton energy density is estimated to be $\gtrsim 10-100 \mathrm{eV} \mathrm{cm}{ }^{-3}$, which is more than 10 times higher than that in the ambient interstellar medium.
\end{abstract}

Keywords: cosmic rays — X-rays: ISM — ISM: supernova remnants

\section{INTRODUCTION}

Supernova remnants (SNRs) are thought to produce Galactic cosmic rays (CRs) via diffusive shock acceleration (DSA). Observational evidence for cosmic-ray acceleration came from ASCA, which detected the non-thermal X-ray emission due to electrons accelerated to the $\mathrm{TeV}$ energy band (Koyama et al. 1995). Recently $\mathrm{GeV}$ and $\mathrm{TeV}$ gammaray observations have revealed that protons and/or electrons are accelerated to energies up to $100 \mathrm{TeV}$ in SNR shells (e.g., Ackermann et al. 2013; Aharonian et al. 2007). In the hadronic origin, the $\mathrm{GeV}$ and $\mathrm{TeV}$ gamma-rays come from decays of $\pi^{0}$ particles, which are produced by interactions between interstellar gas and high-energy cosmic-ray protons above the threshold energy of $280 \mathrm{MeV}$.

In DSA, suprathermal particles (low-energy cosmic rays; LECRs) are accelerated to relativistic energy through multiple crossings of shock front (e.g., Malkov \& Drury 2001). Thus, the energy spectra and fluxes of the LECRs in SNRs provide a key link to generation of the $\mathrm{GeV}$ and $\mathrm{TeV}$ CRs in SNRs. By extrapolating the spectra of high-energy protons obtained by gamma-ray observations (Ackermann et al. 2013) to the low-energy end, we estimate that the (kinetic) energy density of $\mathrm{MeV}$ protons typically amounts to about $1-$ $10 \mathrm{eV} \mathrm{cm}^{-3}$. However, there has been very few observation of LECRs below the MeV band due to lack of an effective

kumiko@cc.nara-wu.ac.jp probe to investigate them.

Observations of $\mathrm{H}_{3}^{+}$absorption lines generated by ionization of $\mathrm{H}_{2}$ molecules have provided information on the cosmic-ray ionization rate (e.g., Indrio \& McCall 2012). While the cross section for $\mathrm{H}_{2}$ ionization by protons peaks at the $\mathrm{keV}$ band, in a dense cloud with the typical column density of molecular hydrogen $N\left(\mathrm{H}_{2}\right)=10^{22} \mathrm{~cm}^{-2}$, the bulk of the ionization is dominated by CRs in the $\mathrm{MeV}-\mathrm{GeV}$ band and followed by those in the keV band (Padovani et al. 2009). Although $\mathrm{H}_{3}^{+}$is produced anywhere with $\mathrm{H}_{2}$ molecules, measurements of the ionization rate require bright infrared sources as a background, and thus there are limited samples of $\mathrm{H}_{3}^{+}$observations yet (Indrio \& McCall 2012). Furthermore, $\mathrm{H}_{3}^{+}$observations cannot provide the information on a spectrum and energy density of LECRs.

Voyager-I traveled 122 astronomical units away from the Earth and observed the spectra of CRs immediately outside the heliosphere down to several $\mathrm{MeV}$ per nucleon without an effect by solar modulation (Stone et al. 2013). Although Voyager-I advances our understanding of LECRs, in order to measure their spectra and energy density in SNRs or the Galactic interstellar space, we should observe LECRs just near the acceleration source because of the short diffusion length of LECRs.

Tatischeff et al. (2012) studied signatures that allow identification of radiation from LECRs in X-ray spectra showing the neutral iron line $(\mathrm{Fe} \mathrm{I} \mathrm{K} \alpha)$ at $6.40 \mathrm{keV}$. By applying the developed models to the X-ray emission from the 
Arches cluster region near the Galactic center, the authors found that the Fe I K $\alpha$ line from the region is likely produced by LECRs. Nobukawa et al. (2015) have also demonstrated that the $\mathrm{Fe} \mathrm{I} \mathrm{K} \alpha$ line can be a unique and powerful probe to investigate LECR protons (LECRp) in the observations of the Galactic disk at $l=1^{\circ} .5-3^{\circ} .5$. When protons in the MeV band collide with molecular clouds (MCs), the Fe I K $\alpha$ line is produced via inner-shell ionization of neutral iron. As for SNRs, there are only two possible samples where the LECRinduced Fe I K $\alpha$ line was detected: Sato et al. (2014, 2016) found from two SNRs (3C 391 and Kes 79) a hint of the Fe I K $\alpha$ line feature, which can be produced by interactions of locally accelerated protons with the surrounding MCs. All the above Fe I K $\alpha$ line emissions but for the Arches cluster were detected by sensitive observations with the X-ray Imaging Spectrometer (XIS) onboard Suzaku, which has advantages of the low and stable background and high sensitivity, especially in the iron K-shell band (Mitsuda et al. 2007; Koyama et al. 2007).

In order to investigate the $\mathrm{Fe} \mathrm{I} \mathrm{K} \alpha$ line in SNRs in our galaxy, we should carefully estimate the X-ray background, which is dominated by the Galactic ridge $\mathrm{X}$-ray emission (GRXE). The distribution and spectrum of the GRXE is well studied in the inner Galactic ridge $\left(|l|<30^{\circ}-40^{\circ},|b|<1^{\circ}\right.$; Nobukawa et al. 2016; Yamauchi et al. 2016). The Fe I K $\alpha$ intensity smoothly distributes in the eastern side although there seems to be a local structure in the western side (figure 2 of Yamauchi et al. 2016). In this paper, we focus on the eastern region of the inner Galactic ridge, and search for the Fe I K $\alpha$ line in seven SNRs from the archives of the Suzaku XIS. We then newly detect the Fe I K $\alpha$ line from five of the SNRs. Based on the spatial and spectral analysis, we discuss the origin of the $\mathrm{Fe} \mathrm{I} \mathrm{K} \alpha$ line. The error bars given in the spectra are at the $1 \sigma$ confidence level.

\section{OBSERVATIONS AND DATA REDUCTION}

In the archive data of the Suzaku XIS, we picked up seven X-ray emitting SNRs which are located in the region of $6^{\circ} \lesssim l \lesssim 40^{\circ},|b|<1^{\circ}$ : W28, Kes 67, Kes 69, Kes 75, Kes 78, 3C 396, and W44. The observation and analysis log is listed in table 1. Three out of the seven samples, W28, Kes 69, and W44, are classified as mixed-morphology SNRs (Rho \& Petre 1998; Jiang et al. 2010), associated with MCs. Accelerated cosmic-ray particles can bombard the MCs to produce the $\mathrm{Fe} \mathrm{I} \mathrm{K} \alpha$ line.

The XIS consists of four X-ray CCD cameras placed on the focal plane of the X-Ray Telescope (XRT; Serlemitsos et al. 2007). The XIS has a field of view (FOV) of $17 . .^{\prime} 8 \times 17 . ' 8$. Three of the sensors (XIS 0, 2, and 3) have front-illuminated (FI) devices, while the other one (XIS 1) contains a backilluminated (BI) device. The whole FOV of XIS 2 and onefourth of XIS 0 have been out of function since 2006 November and 2009 June, respectively, and thus the data from the remaining devices were used.
We reprocessed the data by using xispi in the analysis software package, HEAsoft 6.18.1, and the Suzaku calibration database (CALDB) released in 2016 February, with the standard event selection criteria for the XIS data processing. The response file (arf) and redistribution file ( $\mathrm{rmf}$ ) were produced by xissimarfgen and xisrmfgen (Ishisaki et al. 2007), respectively. The non-X-ray background (NXB) was estimated by xisnxbgen (Tawa et al. 2008).

\section{ANALYSIS AND RESULTS}

\subsection{The iron K-shell line flux from the XIS field}

We made an X-ray spectrum from each FOV of the SNR observations (table 1), excluding point-like sources in the fields. X-ray spectra of all the picked-up SNRs but for Kes 67 have been well studied: the electron temperature of SNR plasma is $\lesssim 1 \mathrm{keV}$ (Sawada \& Koyama 2012; Bocchino et al. 2012; Bamba et al. 2016; Su et al. 2009; Uchida et al. 2012; $\mathrm{Su}$ et al. 2011). Since no previous report is available for Kes 67, we analyzed the full-band spectrum and obtained the temperature of $0.4 \pm 0.1 \mathrm{keV}$. Such low-temperature plasmas are dim above $5 \mathrm{keV}$ band and hardly emit iron K-shell lines.

The seven SNRs are located on the Galactic ridge $\left(6^{\circ} \lesssim l \lesssim 40^{\circ},|b|<1^{\circ}\right)$, and hence the X-ray flux above $5 \mathrm{keV}$ is dominated by the GRXE (Uchiyama et al. 2013; Yamauchi et al. 2016), which has strong K-shell lines of neutral (Fe I K $\alpha$ ), helium-like (Fe XXV He $\alpha$ ), and hydrogen-like iron (Fe XXVI Ly $\alpha$ ) at $6.40 \mathrm{keV}, 6.68 \mathrm{keV}$, and $6.97 \mathrm{keV}$, respectively. The only exception is the FOV containing Kes 75 , which has a pulsar, PSR J1846-0258, and its pulsar wind nebula, (PWN; Helfand et al. 2003). Suzaku XRT cannot spatially resolve the X-ray bright point-like source and the thermal component. Non-thermal emission from the source is not negligible in the $5-8 \mathrm{keV}$ band. However, since the pulsar and PWN show no emission line, it does not affect the iron K-shell line measurement.

We subtracted the NXB and the cosmic X-ray background (CXB) from the spectra. The CXB is expressed as a power law with the photon index of 1.4 and the flux of $8.2 \times 10^{-7}$ photons $\mathrm{cm}^{-2} \mathrm{~s}^{-1} \operatorname{arcmin}^{-2} \mathrm{keV}^{-1}$ at $1 \mathrm{keV}$ (Kushino et al. 2002). We fitted the $4-9 \mathrm{keV}$ band spectra with a phenomenological model consisting of an absorbed power law and four Gaussians. The line energies of the Gaussians are fixed to $6.40,6.68,6.97$, and $7.06 \mathrm{keV}$ (FeI-K $\beta$ ) (Kaastra \& Mewe 1993; Smith et al. 2001). The intensity of the Fe I K $\beta$ line is fixed to 0.125 times that of the Fe I K $\alpha$ line (Kaastra \& Mewe 1993), while those of the other lines are free parameters. Since the absorption has no significant effect on the relevant energy band, absorption column density of the interstellar medium is fixed to $3 \times 10^{22} \mathrm{~cm}^{-2}$ (Uchiyama et al. 2013). We used bremsstrahlung instead of a power law, but the line intensities did not change.

The best-fit intensities of the $\mathrm{Fe}$ I $\mathrm{K} \alpha$ and $\mathrm{Fe}$ XXV $\mathrm{He} \alpha$ lines, and the intensity ratio of $\mathrm{Fe} \mathrm{I} \mathrm{K} \alpha / \mathrm{Fe} \mathrm{XXV} \mathrm{He} \alpha$ in the in- 
Table 1. Observation log and intensities of the Fe I K $\alpha$ and $\mathrm{Fe} \mathrm{XXV} \mathrm{He} \alpha$ lines and the line ratio for each FOV.

\begin{tabular}{|c|c|c|c|c|c|c|c|c|}
\hline \multirow[t]{2}{*}{ Object } & \multirow[t]{2}{*}{ Obs. ID } & \multicolumn{2}{|c|}{ Pointing direction } & \multirow{2}{*}{$\begin{array}{l}\text { Obs. start } \\
\text { (UT) }\end{array}$} & \multirow{2}{*}{$\begin{array}{c}\text { Exposure } \\
(\mathrm{ks})\end{array}$} & \multicolumn{2}{|c|}{ Line intensity ${ }^{\mathrm{a}, \mathrm{b}}$} & \multirow[t]{2}{*}{ Ratio $^{b, c}$} \\
\hline & & $l\left(^{\circ}\right)$ & $b\left(^{\circ}\right)$ & & & $\mathrm{Fe} \mathrm{I} \mathrm{K} \alpha$ & Fe XXV He $\alpha$ & \\
\hline \multirow[t]{5}{*}{ W28 } & 505005010 & 6.47 & -0.00 & 2010 Apr 03 & 73.0 & $2.41 \pm 0.43$ & $11.76 \pm 0.55$ & $0.21 \pm 0.04$ \\
\hline & 505006010 & 6.68 & -0.20 & 2011 Feb 25 & 100.0 & $2.95 \pm 0.38$ & $6.82 \pm 0.42$ & $0.43 \pm 0.06$ \\
\hline & 506036010 & 6.15 & 0.07 & 2011 Oct 10 & 151.1 & $2.29 \pm 0.32$ & $12.86 \pm 0.42$ & $0.18 \pm 0.03$ \\
\hline & 508006010 & 6.38 & -0.24 & 2014 Mar 22 & 40.9 & $3.21 \pm 0.64$ & $8.10 \pm 0.75$ & $0.40 \pm 0.09$ \\
\hline & 508006020 & 6.39 & -0.25 & 2014 Oct 08 & 61.7 & $3.36 \pm 0.50$ & $8.22 \pm 0.57$ & $0.41 \pm 0.07$ \\
\hline Kes 67 & 506051010 & 18.78 & 0.40 & 2012 Mar 08 & 52.0 & $2.74 \pm 0.54$ & $5.98 \pm 0.60$ & $0.46 \pm 0.10$ \\
\hline Kes 69 & 509037010 & 21.82 & -0.57 & 2014 Sep 27 & 77.3 & $3.55 \pm 0.42$ & $5.17 \pm 0.44$ & $0.69 \pm 0.10$ \\
\hline Kes 75 & 404081010 & 29.77 & -0.20 & 2009 Apr 15 & 104.3 & $1.33 \pm 0.56$ & $5.91 \pm 0.61$ & $0.22 \pm 0.10$ \\
\hline \multirow[t]{2}{*}{ Kes 78} & 507035010 & 32.88 & -0.00 & 2012 Apr 20 & 55.1 & $2.56 \pm 0.64$ & $4.82 \pm 0.68$ & $0.53 \pm 0.15$ \\
\hline & 507036010 & 32.69 & -0.08 & 2012 Apr 21 & 52.2 & $1.15 \pm 0.45$ & $5.76 \pm 0.52$ & $0.20 \pm 0.09$ \\
\hline \multirow[t]{6}{*}{ W44 } & 505004010 & 34.70 & -0.41 & 2010 Apr 10 & 61.2 & $2.37 \pm 0.47$ & $3.51 \pm 0.49$ & $0.68 \pm 0.16$ \\
\hline & 508002010 & 34.60 & -0.36 & 2013 Oct 24 & 61.1 & $2.00 \pm 0.53$ & $3.47 \pm 0.57$ & $0.57 \pm 0.18$ \\
\hline & 508003010 & 34.56 & -0.52 & 2013 Oct 22 & 66.7 & $1.95 \pm 0.54$ & $3.26 \pm 0.54$ & $0.60 \pm 0.19$ \\
\hline & 508003020 & 34.56 & -0.51 & 2014 Apr 09 & 32.4 & $1.61 \pm 0.82$ & $3.69 \pm 0.83$ & $0.44 \pm 0.24$ \\
\hline & 508004010 & 34.67 & -0.55 & 2013 Oct 18 & 58.3 & $1.23 \pm 0.44$ & $4.02 \pm 0.51$ & $0.31 \pm 0.11$ \\
\hline & 508005010 & 34.76 & -0.30 & 2013 Oct 19 & 55.7 & $1.48 \pm 0.49$ & $4.27 \pm 0.52$ & $0.35 \pm 0.12$ \\
\hline 3C 396 & 509038010 & 39.19 & -0.30 & 2014 Apr 26 & 82.8 & $1.45 \pm 0.39$ & $5.30 \pm 0.44$ & $0.27 \pm 0.08$ \\
\hline
\end{tabular}

dividual FOVs are summarized in table 1. The Fe XXV He $\alpha$ line intensity in W28 is the highest among the SNRs. This is because W28 is located closest to the Galactic center among the SNRs $\left(l \sim 6^{\circ}, b \sim-0.2^{\circ}\right)$, where the GRXE becomes stronger with decreasing distance from the Galactic center (Uchiyama et al. 2013; Yamauchi et al. 2016). The intensity ratios of $\mathrm{Fe} \mathrm{I} \mathrm{K} \alpha / \mathrm{Fe} \mathrm{XXV} \mathrm{He} \alpha$ from all the fields of the seven SNRs are compared to the averaged one in the GRXE of $0.27 \pm 0.02$ (at the $1 \sigma$ level, see table 3, Nobukawa et al. 2016). The flux ratios of $\mathrm{Fe} I \mathrm{~K} \alpha / \mathrm{Fe} \mathrm{XXV} \mathrm{He} \alpha$ in the FOVs containing W28, Kes 67, Kes 69, Kes 78, and W44 are higher than the average of the GRXE with the $1 \sigma$ level, while those containing Kes 75 and 3C 396 are consistent with the GRXE within $1 \sigma$ errors. Thus, we suspect that the five SNRs, W28, Kes 67, Kes 69, Kes 78, and W44, have enhanced Fe I K $\alpha$ line emissions. We examine the enhancement of the $\mathrm{Fe}$ I K $\alpha$ line emission in detail by spectral analyses in the next subsection.

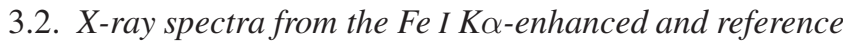 regions}

In order to investigate the $\mathrm{Fe}$ I $\mathrm{K} \alpha$ line enhanced regions of the five SNRs, we made the Fe I K $\alpha$ line band images $(6.2-6.5 \mathrm{keV})$ with the binning size of $80 \times 80$ pixels $\left(1.4 \times 1.4 \operatorname{arcmin}^{2}\right)$ and Gaussian smoothing is applied with $\sigma=2$ bins (Kes 67, Kes 69, Kes78, and W44) or 2.5 bins (W28) as shown in right panels in figure 1. We call the Fe I K $\alpha$ line enhanced region in each SNR as the "enhanced region", while the nearby region is the "reference region", which are indicated by the dashed lines and solid lines, respectively. The enhanced and reference regions have typically $\sim 1.2$ and $\sim 0.6$ photons per bin, respectively. We should note that the figures show rough distributions of the Fe I K $\alpha$ intensity due to the limited statistics.

We also made the continuum band images of the low and high energies, for comparison. Here, we did not use the events of the BI CCD (XIS 1) because of poorer signal-tonoise ratios in the high-energy band. Figures $1 \mathrm{a}-1 \mathrm{e}$ show the XIS images of W28, Kes 67, Kes 69, Kes 78, and W44, respectively. In the soft X-ray band $(0.5-2.0 \mathrm{keV})$, we see clear emission associated with the SNRs, while in the hard $\mathrm{X}$-ray band of 5.0-8.0 keV, no structure accompanied with the soft band is found. Thus, all these SNRs are soft Xray sources with electron temperature of $\lesssim 0.8 \mathrm{keV}$, which are confirmed in Sawada \& Koyama (2012), Bocchino et al. (2012), Bamba et al. (2016), and Uchida et al. (2012) for W28, Kes 69, Kes 78, and W44, respectively. Kes 67 is also a soft X-ray source with an electron temperature of $\sim 0.4 \mathrm{keV}$ 
(a)

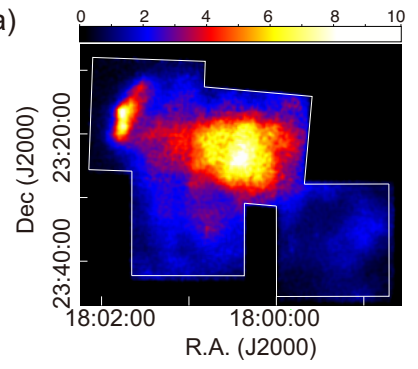

(b)

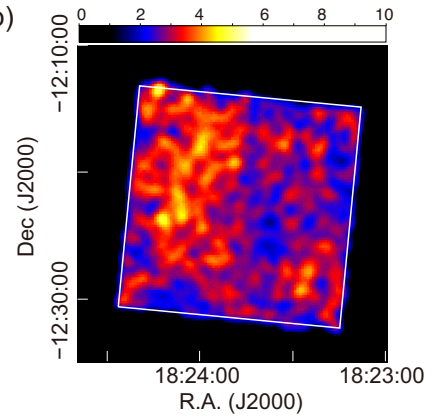

(c)

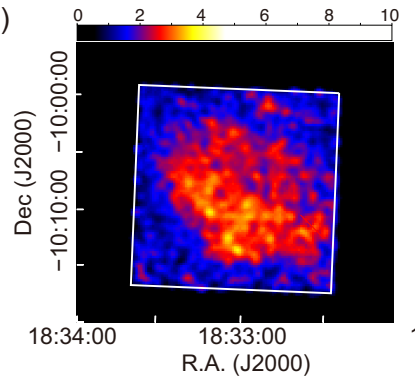

(d)

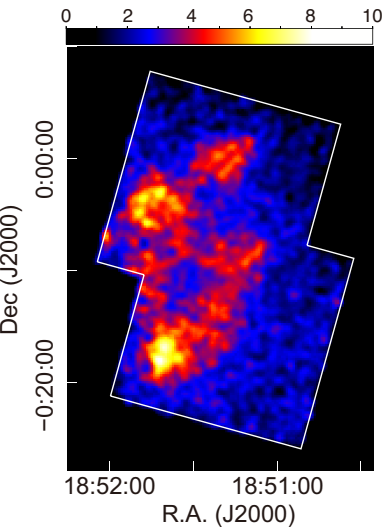

(e)

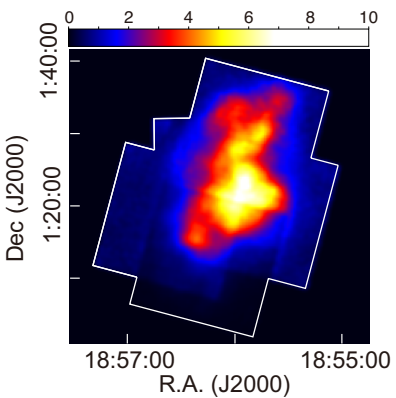

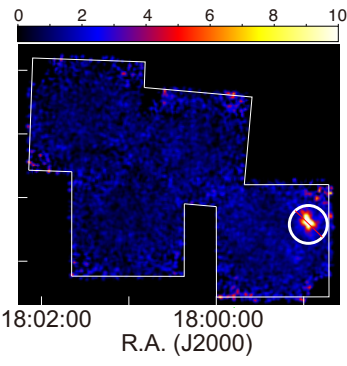
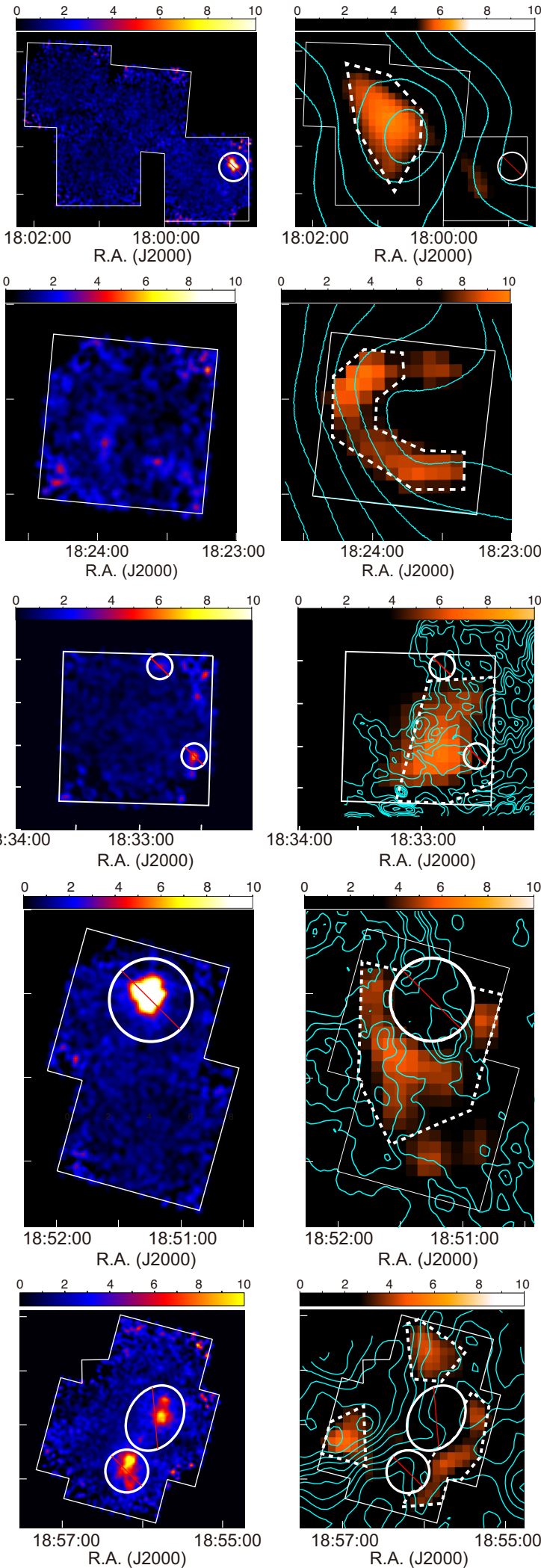

Figure 1. Band images of (a) W28, (b) Kes 67, (c) Kes 69, (d) Kes 78, and (e) W44. The left, center, and right panels show the images of the soft $(0.5-2 \mathrm{keV})$, hard $(5-8 \mathrm{keV})$, and the Fe I K $\alpha$ line band $(6.2-6.5 \mathrm{keV})$, respectively (color bars in arbitrary unit). Vignetting is corrected and the NXB is subtracted. The regions surrounded by the white lines in the individual panels show the FOVs of the XIS. Point-like or slightly extended sources are marked with solid white circle or ellipse regions, which are described in section 3.2. In the right panels, those regions are excluded from the images. The dashed lines in the right panels indicate the "enhanced regions" (see text). The contours in the right panels show the distributions of HI at $37.5 \mathrm{~km} \mathrm{~s}^{-1}$ (Velázquez et al. 2002), HI at $18.1 \mathrm{~km} \mathrm{~s}^{-1}$ (Dubner et al. 1999, the intensity of HI increases towards the east), ${ }^{12} \mathrm{CO}(J=1-0)$ at $80-81 \mathrm{~km} \mathrm{~s}^{-1}$ (Zhou et al. 2009), ${ }^{13} \mathrm{CO}(J=1-0)$ at $80-84 \mathrm{~km} \mathrm{~s}^{-1}$ (Zhou \& Chen 2011), and ${ }^{12} \mathrm{CO}(J=2-1)$ at $40.0-50.3 \mathrm{~km} \mathrm{~s}{ }^{-1}$ (Yoshiike et al. 2013), for (a), (b), (c), (d), and (e), respectively. 
(section 3.1).

In W28, Kes 69, Kes 78, and W44, some point-like or slightly extended sources are found (figures $1 \mathrm{a}, 1 \mathrm{c}, 1 \mathrm{~d}$, and 1e). The point source in the south-west of W28 is reported by Pannuti et al. (2017); the object, CXOU J175857.55233400.3 , is likely a cataclysmic variable or a quiescent lowmass X-ray binary. Two point-like sources located in the north and the west of Kes 69 are reported by Bocchino et al. (2012). However no further information on these sources is available. The bright point source in the north of Kes 78 is 2XMM J185114.3-000004. Bamba et al. (2016) suggested that this object can be a supergiant fast X-ray transient. In the central region of W44, we see two extended sources. The northern source is reported by Uchida et al. (2012). The authors claimed that this hard X-ray emission can be due to a synchrotron source. However, thanks to deep follow-up observations, our quick analysis revealed a hint of an emission line at $\sim 6.15 \mathrm{keV}$ from this hard diffuse source. Thus this source is likely a new identified cluster of galaxies behind the Galactic plane with the redshift of $z \sim 0.09$. The southern source is a PWN associated with PSR B1853+01 (Frail et al. 1996), and was detected in X-rays by ASCA (Harrus et al. 1996).

In the right panels of figure 1, we see that the morphology of the Fe I K $\alpha$ line emission is clearly different from the softband emission. The NXB-subtracted spectra of the enhanced and reference regions for the individual SNRs are shown in figure 2 . We found excess in the $7-8 \mathrm{keV}$ band, which would come from the Fe XXV $\mathrm{He} \beta$, -He $\gamma$, Fe XXVI Ly $\beta$, -Ly $\gamma$, Ni I K $\alpha$, and Ni XXVII He $\alpha$ lines associated with the GRXE (e.g., Nobukawa et al. 2016). We, then, added six Gaussians to the model, and set the centroids to the values found in AtomDB 3.0.2 (http://www.atomdb.org/). The best-fit fluxes of the Fe I K $\alpha$, Fe XXV $\mathrm{He} \alpha$, and Fe XXVI Ly $\alpha$ lines are shown in table 2, and the best-fit models are shown in figure 2.

We found that the Fe I K $\alpha$ line intensity in the enhanced region is stronger than that in the reference region for each SNR. The significance levels are $1.6 \sigma, 2.7 \sigma, 2.9 \sigma, 2.0 \sigma$, and $2.0 \sigma$ for $\mathrm{W} 28$, Kes 67, Kes 69, Kes 78, and W44, respectively. On the other hand, the Fe XXV He $\alpha$ line intensity in the enhanced region is consistent with the reference region within the $1 \sigma$ error range in each SNR. The Fe XXVI Ly $\alpha$ line intensities in the enhanced regions are also consistent with the reference regions within the $1 \sigma$ error range in all the SNRs except Kes 67. In Kes 67, the Fe XXVI Ly $\alpha$ line intensity of the enhanced region is stronger than that of the reference region. However, the poor statistics prevents detailed investigation of this possible Fe XXVI Ly $\alpha$ enhancement.

In order to determine whether the Fe I $\mathrm{K} \alpha$ line from the ensemble of the SNRs is statistically significant, we made the stacked spectra for the enhanced and reference regions of the individual SNRs, as are shown in figure 3a. The two spectra were fitted with the same phenomenological model used for the individual SNRs. The best-fit curves are given in figure $3 \mathrm{a}$, while the best fit parameters of the Fe I $\mathrm{K} \alpha$, Fe XXV He $\alpha$ and Fe XXVI Ly $\alpha$ intensities are listed in table 3. The flux ratio of Fe I K $\alpha$ between the enhanced and reference regions is $2.1 \pm 0.6$, while those of Fe XXV He $\alpha$ and Fe XXVI Ly $\alpha$ are $0.98 \pm 0.13$ and $1.5 \pm 0.8$, respectively (at $90 \%$ confidence levels). Thus, a clear enhancement of the $\mathrm{Fe} \mathrm{I} \mathrm{K} \alpha$ line from the surrounding reference regions is found with more than $4.3 \sigma$ confidence, while no enhancement of Fe XXVI Ly $\alpha$ is found.

We, then, subtracted the stacked spectrum of the reference regions from that of the enhanced regions. Since the GRXE dominates the reference and enhanced spectra, this subtraction should be based on a reliable GRXE estimation in both the spectra. The relevant GRXE may be often contaminated by the stray light of nearby bright sources (Koyama et al. 1989). This contamination in an iron K-shell line is smaller than that in the continuum (e.g., the $5-8 \mathrm{keV}$ band), because the equivalent width (EW; the line flux divided by the continuum flux) of the iron K-shell line in the Galactic bright sources is typically $\lesssim 100 \mathrm{eV}$, which is far smaller than that of the GRXE (e.g., Koyama et al. 1989; Revnivtsev et al. 2006). Thus, assuming that the Fe XXV He $\alpha$ line flux is a good indicator for the flux of the GRXE, we fine-tuned the positional difference of the GRXE flux between the enhanced and reference regions, using the $\mathrm{Fe}$ XXV He $\alpha$ line intensity (see Nobukawa et al. 2015); we made the spectrum of the enhanced emission taking account of the difference of the $\mathrm{Fe}$ XXV $\mathrm{He} \alpha$ line intensity by $1.7 \%$. The result is given in figure $3 \mathrm{~b}$. We fit the obtained spectrum with a power law plus a Gaussian at $6.40 \mathrm{keV}$. The EW and photon index are measured to be $1.0_{-0.4}^{+0.7} \mathrm{keV}$ and $4.1_{-2.4}^{+3.8}$, respectively (at $90 \%$ confidence levels). For a conservative estimate of the lower limit of the $\mathrm{Fe} \mathrm{I} \mathrm{K} \alpha \mathrm{EW}$, we took account of the $90 \%$ confidence error of the Fe XXV He $\alpha$ line intensity. Then, we obtained that the Fe I K $\alpha \mathrm{EW}$ is at least $\sim 400 \mathrm{eV}$. Instead of the analysis of the stacked spectra, we tried subtracting the reference spectrum from the enhanced spectrum for each SNR and fitting the five spectra simultaneously; the result is consistent with the analysis of the stacked spectra.

Since the above stacking process might be a subject of systematic errors, we estimated the possible systematic errors based on simulations in this paragraph. We simulated the spectra from the enhanced and background regions of each SNR, and evaluated the Fe I K $\alpha$ intensities by performing the same stacking analysis as we did for the observational data. In this simulation process, we assumed the exposure time as the actual observations. After 100 trials of the simulations, the distribution of the Fe I K $\alpha$ intensities obtained has a mean of $1.8 \times 10^{-8}$ and a standard deviation of $0.4 \times 10^{-8}$ photons $\mathrm{s}^{-1} \mathrm{~cm}^{-2} \operatorname{arcmin}^{-2}$, which are almost the same as the enhanced Fe I K $\alpha$ line intensity and the statistical error obtained from the observational data. The distribution is shown in figure 4 . We furthermore performed the same simulation 
6
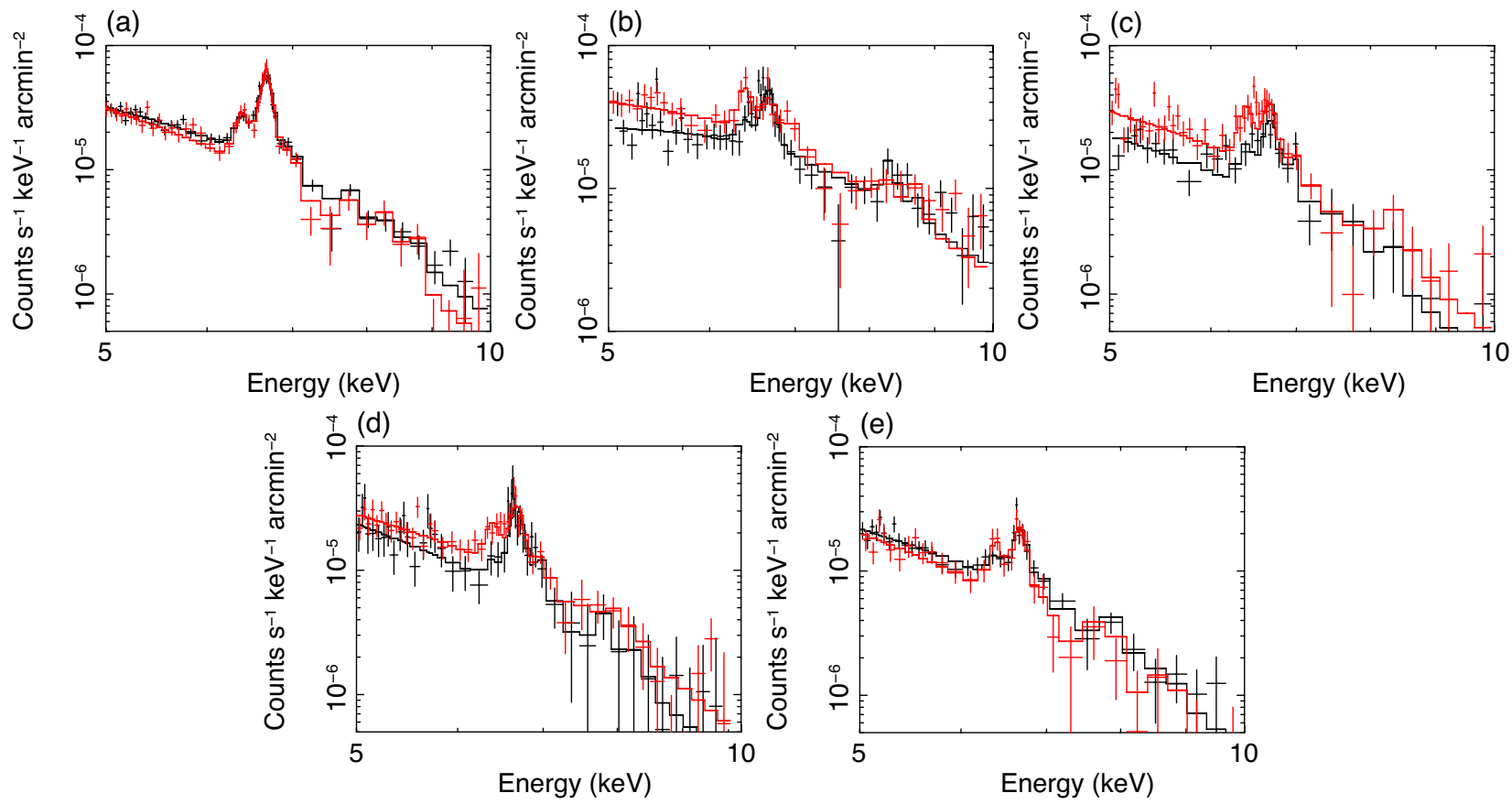

Figure 2. Spectra extracted from the Fe I K $\alpha$ enhanced (red) and reference (black) regions of (a) W28, (b) Kes 67, (c) Kes 69, (d) Kes 78, and (e) W44. The solid lines show the best-fit models consisting of a power law plus ten Gaussians (see text). Errors are quoted at $1 \sigma$ confidence levels.

Table 2. Intensities of the $\mathrm{Fe}$ I K $\alpha$, Fe XXV He $\alpha$, and Fe XXVI Ly $\alpha$ lines and intensity ratios in the enhanced and reference regions.

\begin{tabular}{lcccccccccc}
\hline \hline \multirow{2}{*}{ Object } & \multicolumn{2}{c}{ Fe I K ${ }^{\text {a,b }}$} & \multicolumn{2}{c}{ Fe XXV He $\alpha^{\text {a,b }}$} & \multicolumn{2}{c}{ Fe XXVI Ly ${ }^{\text {a,b }}$} & \multicolumn{3}{c}{ Fe I K $\alpha$ ratio ${ }^{\text {b,c }}$} & \multicolumn{2}{c}{ Fe XXVI Ly $\alpha$ ratio $^{\text {b,d }}$} \\
& Enhanced & Reference & Enhanced & Reference & Enhanced & Reference & Enhanced & Reference & Enhanced & Reference \\
\hline W28 & $3.14 \pm 0.43$ & $2.33 \pm 0.29$ & $9.80 \pm 0.53$ & $9.42 \pm 0.35$ & $1.52 \pm 0.43$ & $1.29 \pm 0.30$ & $0.32 \pm 0.05$ & $0.25 \pm 0.03$ & $0.16 \pm 0.04$ & $0.14 \pm 0.03$ \\
Kes 67 & $6.00 \pm 1.14$ & $2.39 \pm 0.73$ & $5.72 \pm 1.16$ & $7.35 \pm 0.84$ & $3.74 \pm 1.14$ & $<1.15$ & $1.05 \pm 0.29$ & $0.33 \pm 0.11$ & $0.65 \pm 0.24$ & $<0.18$ \\
Kes 69 & $4.35 \pm 0.74$ & $1.57 \pm 0.59$ & $5.44 \pm 0.77$ & $4.38 \pm 0.65$ & $0.76 \pm 0.69$ & $1.24 \pm 0.65$ & $0.80 \pm 0.14$ & $0.36 \pm 0.14$ & $0.14 \pm 0.13$ & $0.28 \pm 0.15$ \\
Kes 78 & $2.62 \pm 0.56$ & $0.81 \pm 0.69$ & $5.19 \pm 0.61$ & $5.66 \pm 0.80$ & $1.33 \pm 0.61$ & $1.62 \pm 0.72$ & $0.50 \pm 0.12$ & $0.14 \pm 0.12$ & $0.26 \pm 0.12$ & $0.29 \pm 0.13$ \\
W44 & $2.18 \pm 0.55$ & $0.92 \pm 0.32$ & $3.66 \pm 0.57$ & $3.47 \pm 0.35$ & $0.96 \pm 0.60$ & $0.91 \pm 0.33$ & $0.60 \pm 0.18$ & $0.27 \pm 0.10$ & $0.26 \pm 0.17$ & $0.26 \pm 0.10$ \\
\hline
\end{tabular}

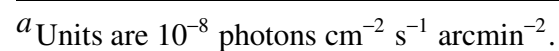

$b_{\text {Errors are quoted at the } 1 \sigma \text { level. }}$

${ }^{c}$ Intensity ratio of $\mathrm{Fe}$ I $\mathrm{K} \alpha / \mathrm{Fe} \mathrm{XXV} \mathrm{He} \alpha$.

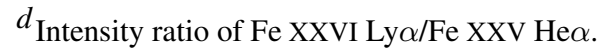

but with 100 times longer exposure assumed. Then, the dispersion became $0.05 \times 10^{-8}$ photons s $\mathrm{sm}^{-1} \mathrm{arcmin}^{-2}$, one order of magnitude smaller than that calculated with the actual exposure. Therefore, we conclude that the stacking process introduces no significant systematic errors which dominate over the statistical errors.

\section{DISCUSSION}

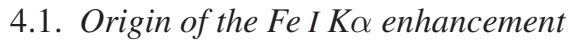

In the region of $6^{\circ} \lesssim l \lesssim 40^{\circ},|b|<1^{\circ}$, we have found the enhanced Fe I K $\alpha$ emission from five SNRs, W28, Kes 67,
Kes 69, Kes 78 and W44. In the same region, Sato et al. (2014, 2016) also found $\mathrm{Fe}$ I K $\alpha$-enhancements from Kes 79 and 3C 391. The origin of the Fe I K $\alpha$ emission would be the same. In comparison with the Fe XXV He $\alpha$ and Fe XXVI Ly $\alpha$ lines, we discuss the origin of the $\mathrm{Fe}$ I $\mathrm{K} \alpha$ line emission from the SNRs.

\subsubsection{Fluctuations of the GRXE?}

The X-ray emission in the $>5 \mathrm{keV}$ band in each SNR is dominated by the GRXE. Is the enhanced Fe I K $\alpha$ emission simply a statistical fluctuation of the GRXE? To answer this question, we list the mean iron $\mathrm{K}$-shell line flux of the 
Table 3. Intensities of the $\mathrm{Fe} I \mathrm{~K} \alpha, \mathrm{Fe} X X V \mathrm{He} \alpha$, and $\mathrm{Fe}$ XXVI Ly $\alpha$ lines and intensity ratios of the integrated spectra of the enhanced and reference regions and the GRXE.

\begin{tabular}{cccccc}
\hline \hline \multirow{2}{*}{ Region } & \multicolumn{3}{c}{ Line intensity $^{\mathrm{a}, \mathrm{b}}$} & \multicolumn{2}{c}{ Intensity ratio $^{\mathrm{b}}$} \\
& Fe I K $\alpha$ & Fe XXV He $\alpha$ & Fe XXVI Ly $\alpha$ & Fe I K $\alpha /$ Fe XXV He $\alpha$ & Fe XXVI Ly $\alpha /$ Fe XXV He $\alpha$ \\
\hline Enhanced & $3.37 \pm 0.54$ & $5.73 \pm 0.56$ & $1.47 \pm 0.53$ & $0.59 \pm 0.11$ & $0.26 \pm 0.10$ \\
Reference & $1.60 \pm 0.42$ & $5.83 \pm 0.48$ & $1.00 \pm 0.43$ & $0.27 \pm 0.08$ & $0.17 \pm 0.08$ \\
\hline GRXE $^{\mathrm{c}}$ & $1.6 \pm 0.2$ & $6.0 \pm 0.3$ & $1.0 \pm 0.2$ & $0.27 \pm 0.03$ & $0.17 \pm 0.03$ \\
\hline
\end{tabular}

$a_{\text {Units are }} 10^{-8}$ photons $\mathrm{cm}^{-2} \mathrm{~s}^{-1} \operatorname{arcmin}^{-2}$.

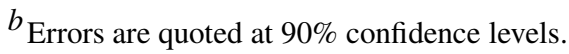

${ }^{c} 10^{\circ}<|l|<30^{\circ}$ and $|b|<1^{\circ}$ (Nobukawa et al. 2016).
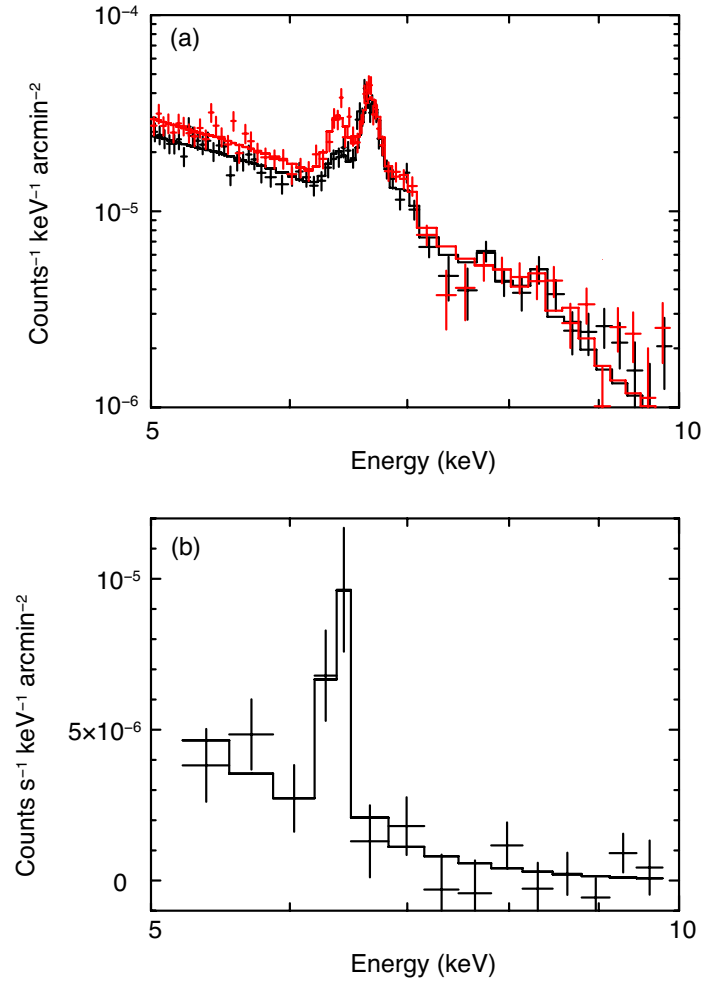

Figure 3. (a) X-ray spectra extracted from the enhanced region (red) and the reference region (black). The red and black lines show the best-fit models consisting of a power law plus ten Gaussians (see text). (b) X-ray spectrum of the enhanced emission, which is obtained by subtracting the spectrum of the reference region from that of the enhanced region (see text). Errors are quoted at $1 \sigma$ confidence levels.

GRXE $\left(|l|=10^{\circ}-30^{\circ}\right)$ in table 3, following Nobukawa et al. (2016). In table 3, the line fluxes of $\mathrm{Fe} \mathrm{I} \mathrm{K} \alpha$, Fe XXV He $\alpha$ and Fe XXVI Ly $\alpha$ in the reference region agree to those of the mean GRXE. Also the Fe XXV He $\alpha$ and Fe XXVI Ly $\alpha$ line fluxes in the enhanced region agree with those of the mean GRXE. As is noted in section 3.2, the Fe XXV He $\alpha$ line flux is a more reliable indicator of the GRXE flux than the $5-8 \mathrm{keV}$ flux. We, therefore, use the flux ratios of

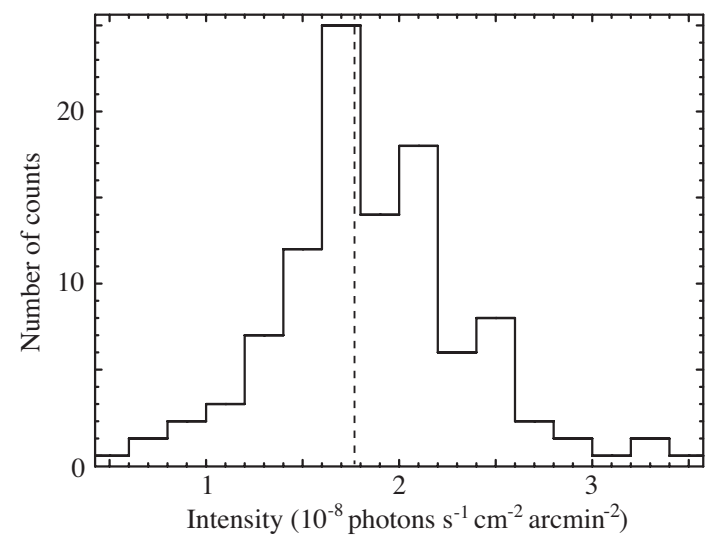

Figure 4. Distribution of the enhanced Fe I K $\alpha$ intensities obtained from 100 trials of the simulations. The distribution has a mean value and a standard deviation of $1.8 \times 10^{-8}$ and $0.4 \times 10^{-8}$ photons $\mathrm{s}^{-1} \mathrm{~cm}^{-2} \operatorname{arcmin}^{-2}$, respectively. The dashed line indicates the best-fit value $\left(1.77 \times 10^{-8}\right.$ photons s $\left.\mathrm{sm}^{-1} \mathrm{arcmin}^{-2}\right)$ obtained with the actual observation data (table 3 ).

Fe I K $\alpha / \mathrm{Fe}$ XXV He $\alpha$ instead of the EW for estimation of the GRXE contribution. The flux ratios of the iron K-shell lines between the enhanced and reference region are also listed in table 3. From table 3, we conclude that the Fe I K $\alpha$ line enhancements are not due to statistical fluctuation of the GRXE, but associated with the SNRs.

\subsubsection{Thermal plasma origin?}

The iron K-shell line emission has been detected in many SNRs. Most of the iron is of the ejecta origin, and hence is located around the center of a supernova explosion. The ironrich ejecta are heated by the reverse shock. Therefore, the ionization state is often lower than helium-like iron in young or middle-aged SNRs, which is called an ionizing plasma (IP). The iron $\mathrm{K} \alpha$ centroids depend on the ionization age, and hence some young SNRs show a very low ionization state of iron with the line centroids of $\sim 6.4 \mathrm{keV}$ (e.g., RCW 86, SN 1006; Yamaguchi et al. 2008a,b). One may argue that the observed $6.40 \mathrm{keV}$ line in the soft X-ray SNRs of our sample is also due to IP. However, with the following two reasons, 
we interpret that the $6.40 \mathrm{keV}$ enhancement would not be of the IP origin.

One is that the morphology of the $6.40 \mathrm{keV}$ emission is clearly different from that of the soft-energy band, the distribution of the SNR plasma. In particular, in Kes 67 and W44, the distributions of the $6.40 \mathrm{keV}$ emission are not centerfilled, like the image of the soft-energy band, but have shelllike structures. This may indicate that the $6.40 \mathrm{keV}$ emission does not arise from the SNR plasma, although the different morphologies do not necessarily mean a different origin.

The other reason is that all the SNRs with the $6.40 \mathrm{keV}$ line enhancements interact with MCs (Claussen et al. 1997; Tian et al. 2007; Zhou et al. 2009; Koralesky et al. 1998; Hoffman et al. 2005), which indicates that these SNRs are likely core-collapse SNRs. Yamaguchi et al. (2014) reported the iron $\mathrm{K} \alpha$ centroids of core-collapse SNRs are significantly higher than $6.40 \mathrm{keV}$. This is in a sharp contrast to Type Ia SNRs, which show lower energy of $<6.5 \mathrm{keV}$. Since the ionization or excitation rate of iron $\mathrm{K}$-shell electrons decreases very rapidly below the electron temperature of $1 \mathrm{keV}$, the iron $\mathrm{K}$-shell line should be detected in SNRs with the electron temperature higher than $\sim 1 \mathrm{keV}$ (Yamaguchi et al. 2014). In other words, it is almost impossible for the SNRs in our sample to emit a detectable iron K-shell line because of the low electron temperature.

\subsubsection{Ionization by $X$-rays or cosmic-ray particles?}

Another scenario is that a bright X-ray source is located near each SNR and X-rays from the source irradiate the gas surrounding the SNR to produce the Fe I K $\alpha$ line. Assuming a typical value for the hydrogen column density (of the ambient gas) $N_{\mathrm{H}}=3 \times 10^{22} \mathrm{~cm}^{-2}$ and a typical Fe I K $\alpha$ enhanced area of $10^{2}-15^{2} \operatorname{arcmin}^{2}$, the source luminosity should be $L_{\mathrm{X}} \sim 1 \times 10^{38}(D / 100 \mathrm{pc})^{2} \mathrm{erg} \mathrm{s}^{-1}$ (Sunyaev \& Churazov 1998), where $D$ is the distance of the Fe I K $\alpha$ line emitting region from the irradiating source. The required luminosity is close to the Eddington luminosity of a neutron star $L_{\text {Edd }}=2 \times 10^{38} \mathrm{erg} \mathrm{s}^{-1}$. Such a bright source is not located nearby the SNRs. Thus the X-ray irradiation scenario is also difficult.

The most plausible scenario is inner-shell ionization of iron atoms in the surrounding gas by CRs accelerated in the SNRs. The EW of the Fe I K $\alpha$ line depends on the irradiating particle and the iron abundance. Here we assume the iron abundance of 1 solar. In the case of the proton bombardment, the EW is around or higher than $\sim 500 \mathrm{eV}$, while in the case of the electron bombardment, the EW is below $400 \mathrm{eV}$ due to strong bremsstrahlung, regardless of the spectral index of the particles (Dogiel et al. 2011). The lower limit of the measured EW of $400 \mathrm{eV}$ rejects the electron origin. The Fe I K $\alpha$ line enhancements in the five SNRs would be due to interactions between LECRp and the ambient cold gas.

\subsection{Implication for the origin of the GRXE}

Nobukawa et al. (2016) reported that the mean spectrum of the GRXE shows significant enhancements of the Fe I $\mathrm{K} \alpha$, Fe XXV $\operatorname{He} \alpha$, and Fe XXVI Ly $\alpha$ lines from the assembly of the known point sources, cataclysmic variables and $\mathrm{X}$ ray active binary stars. On the other hand, in general, the point sources explain the continuum of the GRXE spectrum. Therefore, the other candidates emitting iron lines are required. As is discussed in section 4.1, the excess Fe I K $\alpha$ intensity over the mean GRXE is found in our SNR samples. Since the hard X-ray fluxes $(5-8 \mathrm{keV})$ of the SNRs are negligibly small due to their low temperature of $\lesssim 1 \mathrm{keV}$, these SNRs may not contribute to the GRXE in the 5-8 keV band. Therefore, the candidate sources of the Fe I K $\alpha$ excess in the GRXE are LECRp colliding with cold gas in SNRs. The Fe I K $\alpha$ line excess would be more common among SNRs that are faint in hard X-ray or are not detected in X-ray.

Such SNRs may also have some contribution to the excess of the Fe XXV He $\alpha$ and Fe XXVI Ly $\alpha$ lines in the GRXE above the predicted point-source contribution. Some of the SNRs that show the Fe I K $\alpha$-enhanced emission are known to be have recombining plasma (RP) for silicon and sulfur (W28, W44, and 3C 391; Sawada \& Koyama 2012; Uchida et al. 2012; Sato et al. 2014). Since LECRp can ionize helium-like silicon and sulfur in the hot plasma of SNRs to hydrogen-like ions, they may contribute to production of RP.

LECRp may also generate helium-like and hydrogen-like ions of iron. We roughly estimate ionization rate of heliumlike iron for the cases where iron is ionized by LECRp or thermal electrons in the plasma with $k T \sim 1 \mathrm{keV}$. The ionization rate is proportional to the LECRp/electron density, velocity, and the cross section. Number density of the $\mathrm{MeV}$ protons obtained in this study is $n_{\mathrm{p}} \sim 10^{-5} \mathrm{~cm}^{-3}$ (equivalent to the energy density of $100 \mathrm{eV} \mathrm{cm}^{-3}$; see section 4.4). In the thermal plasma, electrons with energy above the K-shell ionization energy of iron are $\sim 0.01 \%$ of the total; assuming the total electron density of $1 \mathrm{~cm}^{-3}$, we obtain $n_{\mathrm{e}} \sim 10^{-4} \mathrm{~cm}^{-3}$. Ionization cross section of helium-like iron for electrons with the kinetic energy of $\sim 10 \mathrm{keV}$ is $\sigma_{e} \sim 10^{-22} \mathrm{~cm}^{-2}$, which is the same in the order of magnitude as that for LECRp $\left(\sigma_{\mathrm{p}}\right)$ with the kinetic energy of $\sim 10 \mathrm{MeV}$ (Kocharov et al. 2000). Also velocities of electrons $\left(v_{\mathrm{e}}\right)$ and LECRp $\left(v_{\mathrm{p}}\right)$ are similar to each other $(\sim 0.2 c)$. Thus, the ratio of the ionization rate between the cases of LECRp and thermal electrons are $\left(n_{\mathrm{p}} v_{\mathrm{p}} \sigma_{\mathrm{p}}\right) /\left(n_{\mathrm{e}} v_{\mathrm{e}} \sigma_{\mathrm{e}}\right) \sim 0.1$. The LECRp can contribute to production of RP.

For example, a soft X-ray SNR, IC443 has RP emitting the Fe XXV He $\alpha$ and Fe XXVI Ly $\alpha$ lines (Ohnishi et al. 2014). Although the mechanism is under discussion, LECRp may contribute. At present there are limited samples of the hard X-ray faint SNRs with the Fe XXV He $\alpha$ and Fe XXVI Ly $\alpha$ excess, but if the number of such samples increases by further observations, we will possibly be able to evaluate their contribution to the GRXE. 


\subsection{Origin of the MeV protons}

The Fe I K $\alpha$ line emission is expected to be associated with dense gas such as MCs and H I clouds. Therefore, we compared the distribution of the Fe I $\mathrm{K} \alpha$ line emission with that of $\mathrm{H}$ I clouds for W28 and Kes 67, and with that of the CO intensity for Kes 69, Kes 78, and W44. All the five SNRs have been well studied by radio observations. The velocities (LSR) where the individual SNRs are associated with molecular clouds were identified by molecular line broadening (all the SNRs), $1720 \mathrm{MHz}$ OH maser (W28, Kes 69, Kes 78, and W44), and a high line intensity ratio ${ }^{12} \mathrm{CO}(J=2-1) /{ }^{12} \mathrm{CO}(J=1-0)(\mathrm{Kes} 67$ and W44) as well as morphological association (Jiang et al. 2010, and references therein; Zhou \& Chen 2011). The velocities of the H I and $\mathrm{CO}$ intensity maps in figure 1 are consistent with those velocities. In W28, Kes 67, Kes 69, and W44, we found association between the Fe I K $\alpha$ line and dense gas clouds interacting with each SNR (figure 1). The Larmor radius of LECRp is extremely short $\left(\sim 10^{11} \mathrm{~cm}\right.$ at $10 \mathrm{MeV}$ in the magnetic field of $1 \mu \mathrm{G}$ ). Therefore, the Fe I K $\alpha$ line should be emitted just near the LECRp acceleration sites.

Nobukawa et al. (2015) reported that diffuse Fe I K $\alpha$ line emission was discovered in a region $3^{\circ}$ eastern region of the Galactic center. The authors claimed that the Fe I K $\alpha$ line emission is generated by LECRp. Because of the short diffusion length of LECRp, the protons should be produced in situ. There is no SNR in the region. The Fe I K $\alpha$ line emission is considered to be associated with a giant molecular cloud, Bania's Clump 2 exhibiting a large velocity dispersion $\sim 100 \mathrm{~km} \mathrm{~s}^{-1}$ (Bania 1977; Torii et al. 2010). The authors claimed that the LECRp can be produced by stochastic acceleration via alfvénic turbulence. In our case, on the other hand, there is no molecular cloud with such a large velocity dispersion, and hence the same scenario cannot be applied. The most possible scenario is that the $\mathrm{MeV}$ protons come from the SNRs. The spatial association between the Fe I K $\alpha$ line emission and the dense clouds suggests that the $\mathrm{MeV}$ protons are produced by shocks interacting with the dense clouds.

Since the LECRp would be a seed particle to the high energy acceleration up to the $\mathrm{GeV}$ and $\mathrm{TeV}$ energy, we compared the distribution of the Fe I K $\alpha$ line with those of the high energy ( $\mathrm{GeV}$ and $\mathrm{TeV}$ ) gamma rays. The gamma-ray results are available only for W28 and W44; figures 5a and $5 \mathrm{~b}$ present the $\mathrm{TeV}$ and $\mathrm{GeV}$ gamma-ray contours of $\mathrm{W} 28$ and W44, respectively, overlaid on the Fe I K $\alpha$ line band images. The peak of the TeV gamma-ray distribution of W28 lies to the east by $\sim 15^{\prime}$ from the Fe I K $\alpha$ line emission and is located on the FOV edge of Suzaku, where the observing efficiency is poor. The gamma-ray emission is positionally associated with MCs (Aharonian et al. 2008). Since the LECRp cannot penetrate into the $\mathrm{MC}$, this image is not inconsistent with that the $\mathrm{MeV}$ protons are the origin of $\mathrm{TeV}$ protons. The
Fe I K $\alpha$ line emission far from the $\mathrm{TeV}$ emission would be due to low $N_{\mathrm{H}}$ region ( $\sim$ several times of $10^{22} \mathrm{~cm}^{-2}$ ) of neutral gas. The $\mathrm{GeV}$ gamma rays in $\mathrm{W} 44$ are interpreted to be produced via interactions between $\mathrm{GeV}$ protons and the MCs (Abdo et al. 2010; Giuliani et al. 2010). The Fe I K $\alpha$ line could be generated from the low-energy protons accelerated in the $\mathrm{GeV}$ proton region. The $\mathrm{GeV}$ gamma-ray and Fe I $\mathrm{K} \alpha$ line regions well overlap, in spite of the extremely small path length of $\mathrm{MeV}$ protons (the Fe I K $\alpha$ region) compared to the large one of $\mathrm{GeV}$ protons (the $\mathrm{GeV}$ gamma-ray region). This would indicate that the $\mathrm{Fe} \mathrm{I} \mathrm{K \alpha}$ region is located just in front of or behind the gamma-ray region.

\subsection{Energy density of protons}

In the same manner as in Nobukawa et al. (2015), we estimated the energy density of LECRp. The hydrogen column densities of the MCs associated with the SNRs range from $\sim 5 \times 10^{21}$ to $\sim 3 \times 10^{22} \mathrm{~cm}^{-2}$ (Fukui et al. 2008; Tian et al. 2007; Zhou et al. 2009; Zhou \& Chen 2011; Yoshiike et al. 2013). Although the Fe I K $\alpha$ enhancements are not always consistent with the MCs (see section 4.3), we assume these values as the upper limit of the hydrogen column densities of the ambient gas associated with the SNRs, and obtain the lower limit of the proton energy density. If we assume a mono energetic distribution, the lower limit of the proton energy density at $10 \mathrm{MeV}$, where the cross section has a peak of $1.3 \times 10^{-26} \mathrm{~cm}^{2}$ hydrogen-atom ${ }^{-1}$ in solar abundance (Tatischeff et al. 2012; Dogiel et al. 2011; Lodders 2003), is estimated to be $\sim 10-100 \mathrm{eV} \mathrm{cm}^{-3}$. This value is comparable to or one order magnitude higher than the extrapolation of the high-energy CRs (see section 1).

\subsection{Future works}

Among the active X-ray astronomy satellites, XMMNewton can be used to separate the Fe I $\mathrm{K} \alpha$ line (e.g. Tatischeff et al. 2012). We plan combination analysis of Suzaku and XMM-Newton to strengthen the LECR scenario. Moreover, in future X-ray missions, we will be able to perform spectroscopy with a high-energy resolution $(\sim \mathrm{eV})$ and to measure the Fe I K $\alpha$ line much more sensitively.

\section{CONCLUSIONS}

We found the Fe I $\mathrm{K} \alpha$ line from the five SNRs, W28, Kes 67, Kes 69, Kes 78, and W44 among seven samples located in the region of $6^{\circ}<l<40^{\circ}$ and $|b|<1^{\circ}$ observed by Suzaku. The X-ray spectrum of the Fe I K $\alpha$ excess has a large EW ( $>400 \mathrm{eV}$ ) and is well explained by interactions between LECRp, or MeV protons, and the ambient cold gas. The energy density of the LECRp is estimated to be at least $10-100 \mathrm{eV} \mathrm{cm}^{-3}$.

We thank all the members of the Suzaku team. We are grateful to Ping Zhou for providing the $\mathrm{CO}$ data shown 
(a)

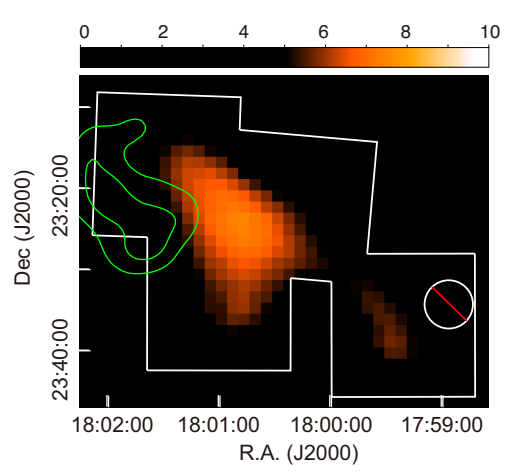

(b)

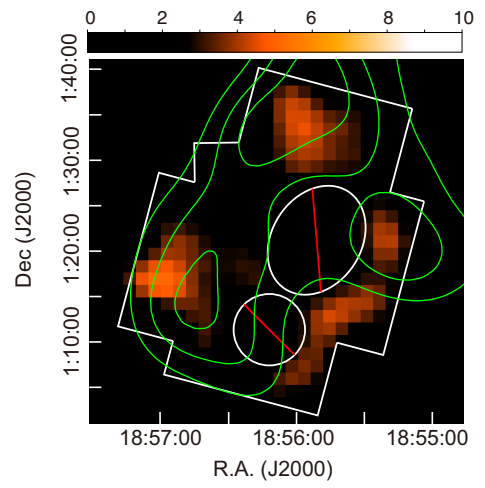

Figure 5. Distributions of the Fe I K $\alpha$ band (same as the right panels of figures 1a and 1e) overlaid with contours of (a) TeV gamma-ray emission for W28 (Aharonian et al. 2008) and (b) gamma-ray emission in the 2-10 GeV band which is observed by Fermi LAT (Abdo et al. 2010) for W44.

in figure 1d. K.K.N. is supported by Research Fellowships of JSPS for Young Scientists. This work was supported by JSPS and MEXT KAKENHI Grant Numbers
JP16J00548 (K.K.N.), JP17K14289 (M.N.), JP25109004 (T.T.), and JP26800102 (H.U.).

\section{REFERENCES}

Abdo, A. A., Ackermann, M., Ajello, M., et al. 2010, Sci, 327, 1103 Ackermann, M., Ajello, M., Allafort, A., et al. 2013, Sci, 339, 807

Aharonian, F., Akhperjanian, A. G., Bazer-Bachi, A. R., et al. 2007, A\&A, 464,235

Aharonian, F., Akhperjanian, A. G., Bazer-Bachi, A. R., et al. 2008, A\&A, 481, 401

Bamba, A., Terada, Y., Hewitt, J., et al. 2016, ApJ, 818, 63

Bania, T. M. 1977, ApJ, 216, 381

Bocchino, F., Bykov, A. M., Chen, Y., et al. 2012, A\&A, 541, A152

Claussen, M. J., Frail, D. A., Goss, W. M., \& Gaume, R. A. 1997, ApJ, 489, 143

Dogiel, V., Chernyshov, D., Koyama, K., Nobukawa, M., \& Cheng, K.-S. 2011, PASJ, 63, 535

Dubner, G., Giacani, E., Reynoso, E., et al. 1999, ApJ, 118, 930

Fukui, Y. and NANTEN team, 2008, in AIP Conf. Proc. 1085, High Energy Gamma-Ray Astronomy, ed. F. A. Aharonian, W. Hofmann, \& F. Rieger (Melville, NY: AIP), 104

Frail, D. A., Giacani, E. B., Goss, W. M., \& Dubner, G. 1996, ApJL, 464, L165

Giuliani, A., Tavani, M., Bulgarelli, A., et al. 2010, A\&A, 516, L11

Harrus, I. M., Hughes, J. P., \& Helfand, D. J. 1996, ApJL, 464, L161

Helfand, D. J., Collins, B. F., \& Gotthelf, E. V. 2003, ApJ, 582, 783

Hoffman, I. M., Goss, W. M., Brogan, C. L., \& Claussen, M. J. 2005, ApJ, 627,803

Indrio, N., \& McCall, B. J. 2012, ApJ, 745, 91

Ishisaki, Y., Maeda, Y., Fujimoto, R., et al. 2007,PASJ, 59, S113

Jiang, B., Chen, Y., Wang, J., et al. 2010, ApJ, 712, 1147

Kaastra, J. S., \& Mewe, R. 1993, A\&A, 97, 443

Kocharov, L., Kovaltsov, G. A., Torsti, J., \& Ostryakov, V. M. 2000, A\&A, 357,716

Koralesky, B., Frail, D. A., Goss, W. M., Claussen, M. J., \& Green, A. J. 1998, ApJ, 116, 1323

Koyama, K., Awaki, H., Kunieda, H., Takano, S., \& Tawara, Y. 1989, Natur, 339, 603

Koyama, K., Petre, R., Gotthelf, E. V., et al. 1995, Natur, 378, 255

Koyama, K., Tsunemi, H., Dotani, T., et al. 2007, PASJ, 59, S23

Kushino, A., Ishisaki, Y., Morita, U., et al. 2002, PASJ, 54, 327

Lodders, K. 2003, ApJ, 591, 1220

Malkov, M. A. and Drury, L. O'C. 2001, RPPh, 64, 429
Mitsuda, K., Bautz, M., Inoue, H., et al. 2007, PASJ, 59, S1

Nobukawa, K. K., Nobukawa, M., Uchiyama, H., et al. 2015, ApJL, 807, L10

Nobukawa, M., Uchiyama, H., Nobukawa, K. K., Yamauchi, S., \& Koyama, K. 2016, ApJ, 833, 268

Ohnishi, T., Uchida, H., Tsuru, T. G., et al. 2014, ApJ, 784, 74

Padovani, M., Galli, D., \& Glassgold, A. E. 2009, A\&A, 501, 619

Pannuti, T. G., Rho, J., Kargaltsev, O., et al. 2017, ApJ, 839, 59

Revnivtsev, M., Molkov, S., \& Sazonov, S. 2006, MNRAS, 373, L11

Rho, J., \& Petre, R. 1998, ApJ, 503, L167

Sato, T., Koyama, K., Takahashi, T., Odaka, H., \& Nakashima, S. 2014, PASJ, 66, 124

Sato, T., Koyama, K., Lee, S.-H., \& Takahashi, T. 2016, PASJ, 68, S8

Sawada, M., \& Koyama, K. 2012, PASJ, 64, 81

Serlemitsos, P. J., Soong, Y., Chan, K.-W., et al. 2007, PASJ, 59, S9

Smith, R. K., Brickhouse, N. S., Liedahl, D. A., \& Raymond, J. C. 2001, ApJ, 556, L91

Stone, E. C., Cummings, A. C., McDonald, F. B., et al. 2013, Sci, 341, 150

Su, Y., Chen, Y., Yang, J., Koo, B.-C., et al. 2009, ApJ, 694, 376

Su, Y., Chen, Y., Yang, J., Koo, B.-C., et al. 2011, ApJ, 727, 43

Sunyaev, R., \& Churazov, E. 1998, MNRAS, 297, 1279

Tatischeff, V., Decourchelle, A., \& Maurin, G. 2012, A\&A, 546, A88

Tawa, N., Hayashida, K., Nagai, M., et al. 2008, PASJ, 60, S11

Tian, W. W., Leahy, D. A., \& Wang, Q. D. 2007, A\&A, 474, 541

Torii, K., Kudo, N., Fujishita, M., et al. 2010, PASJ, 62, 1307

Uchida, H., Koyama, K., Yamaguchi, H., et al. 2012, PASJ, 64, 141

Uchiyama, H., Nobukawa, M., Tsuru, T. G., \& Koyama, K. 2013, PASJ, 65,19

Velázquez, P. F., Dubner, G. M., Goss, W. M., \& Green, A. J. 2002, ApJ, 124,2145

Yamaguchi, H., Koyama, K., Katsuda, S., et al. 2008, PASJ, 60, S141

Yamaguchi, H., Koyama, K., Nakajima, H., et al. 2008, PASJ, 60, S123

Yamaguchi, H., Badenes, C., Petre, et al. 2014, ApJL, 785, L27

Yamauchi, S., Nobukawa, K. K., Nobukawa, M., Uchiyama, H., \& Koyama, K. 2016, PASJ, 68, 59

Yoshiike, S., Fukuda, T., Sano, H., et al. 2013, ApJ, 768, 179

Zhou, X., Chen, Y., Su, Y., \& Yang, J. 2009, ApJ, 691, 516

Zhou, P., \& Chen, Y. 2011, ApJ, 743, 4 